\title{
Free Convection in a Rotating Medium
}

\author{
Michael R. Samuels* \\ Department of Chemical and Metallurgical Engineering, The University of Michigan \\ Ann Arbor, Michigan
}

(Received 15 September 1967; final manuscript received 14 June 1968)

\begin{abstract}
Numerical techniques have been used to study natural convection in a fluid subjected to axial rotation. Axisymmetrical flows were studied in two different geometries for a variety of rotational speeds. For case I $\left(R_{1}=1.0, R_{2}=2.5, H=3.0\right)$, the computed flow patterns were qualitatively compared with those which would be expected from the basic rules of physics, and good agreement was obtained. Rotational speeds between $\Omega=0$, and $\Omega=20$ were studied for a Grashoff number of 3000 . Increased rotational speed was found to decrease the over-all heat transfer rate. For case II $\left(R_{1}=1.0, R_{2}=4.0, H=1.0\right)$, rotational speeds of $\Omega=0.0$ to $\Omega=40.0$ were studied at a Grashoff number of 400 . At low rotational speeds, a single convection cell was found, however, at higher rotational speeds, the single cell became unstable, and changed to a two-celled pattern. This transition resulted in a slight increase in the over-all heat transfer rate.
\end{abstract}

\section{INTRODUCTION}

As early as 1884, Vettin ${ }^{1}$ reported experiments on the convection patterns of a centrally cooled fluid in a rotating basin. Later, $\mathrm{Fultz}^{2}$ reported his studies of water in a centrally cooled rotating pan. He observed both axially symmetrical, and unsymmetrical, but regular, convection patterns. The pan used in these experiments was rather shallow because he was mainly interested in understanding the large scale motions of the atmosphere.

In an attempt to develop an understanding of the origin of terrestrial magnetism, Hide ${ }^{3}$ performed experiments similar to those of Fultz, but in much deeper cylinders, and also observed patterns similar to his. As Hide increased the rotational speed in his experiments, he noted the formation of more and more convection "petals."

Although $\mathrm{Kuo}^{4}$ has made an excellent analysis of the type of instability observed by Fultz and Hide (i.e., the transition from one convection mode to another), some simplifying assumptions were necessary. This paper, and a recent paper by Williams, ${ }^{5}$ represent an attempt to solve the equations governing the flows studied by these authors numerically. No simplifying assumptions, other than the Boussinesq approximations, have been made. As

* Present address: Department of Chemical Engineering, University of Delaware, Newark, Delaware.

1 F. Vettin, Z. Meteorol. 1, 227, 271 (1884).

2 D. Fultz, in Proceedings of the First Symposium on the Use of Models in Geophysics and Fluid Dynamics (U. S. Printing Office, Washington, D. C., 1953), p. 27.

${ }^{3} \mathrm{R}$. Hide, in Proceedings of the First Symposium on the Use of Models in Geophysics and Fluid Dynamics (U. S. Printing Office, Washington, D. C., 1953), p. 101.

${ }^{4}$ H. L. Kuo, in Proceedings of the First Symposium on the Use of Models in Geophysics and Fluid Dynamics (U. S. Printing Office, Washington, D. C., 1953), p. 65.

${ }_{5}^{5}$ G. P. Williams, J. Atmospheric Sci. 24, 144, 161 (1967). a beginning, only axially symmetrical convection is considered. It is hoped that in future studies axially unsymmetrical flows will be studied at ever higher rotational speeds.

\section{MATHEMATICAL DESCRIPTION OF THE PROBLEM}

The model illustrated in Fig. 1 was used as the basis for this study of free convection in a rotating medium.

The central core $r \leq r_{1}$ is assumed to be solid and is held at a temperature $\theta=\theta_{l}$. The outer boundary at $r=r_{2}$ is held at constant temperature $\theta=\theta_{h}$. The bottom wall of the chamber at $z=h$ is a rigid surface, while the upper boundary $z=0$ is assumed to be a free surface. The upper and lower surfaces are assumed to be perfectly insulated against heat losses. Thus, the fluid is confined within the annular region $r_{1} \leq r \leq r_{2}, 0 \leq z \leq h$. The solid boundaries of the chamber are subjected to a constant angular rotation, and it is assumed that no slip occurs on the solid boundaries.

Previous experimental investigation ${ }^{2,3}$ indicated that the flows which developed in this geometry at low rotational speeds were axially symmetrical. However, at higher rotational speeds this symmetrical behavior broke down and a distinct variation of flow pattern with angular position formed.

In this study, numerical techniques are used to solve the Boussinesq approximations to the equations of motion and heat diffusion for axially symmetrical flows. Although the methods presented herein may be extended to cover nonaxially symmetrical flows, ${ }^{6}$ these calculations would have required considerably

\footnotetext{
${ }^{6}$ K. Aziz, Ph.D. thesis, Rice University (1965).
} 
more computing time than was available at present. It is hoped that the advent of higher-speed computers, and better computing techniques for threedimensional flows will remove this restriction in the near future.

The following dimensionless variables are defined:

$$
\begin{array}{rlrl}
U & =\frac{u}{\nu / r_{1}}, & T=\frac{\theta-\theta_{11}}{\theta_{h}-\theta_{i}}, & R=\frac{r}{r_{1}}, \\
V=\frac{v}{\nu / r_{1}}, & P=\frac{p^{1}}{\rho_{i} \nu^{2} / r_{1}^{2}}, & Z=\frac{z}{r_{1}}, \\
W=\frac{w}{\nu / r_{1}}, & \tau=\frac{t}{r_{1}^{2} / \nu} . &
\end{array}
$$

Where $u, w$, and $v$ are radial $r$, vertical $z$, and tangential components of velocity; $k$ is the thermal diffusivity of the fluid; $\theta$ its temperature at any point, and $\beta$ the coefficient of volumetric expansivity $(\beta=-1 / \rho \partial \rho / \partial \theta) . p^{1}$ is the actual pressure at any point minus the hydrostatic pressure in the quiescent state. That is,

$$
p^{1}=p-\left(p_{0}+\rho g z\right) .
$$

The dimensionless form of the Boussinesq approximations to the equations of motion and energy are then written as

$$
\begin{gathered}
\frac{\partial U}{\partial \tau}+U \frac{\partial U}{\partial R}+W \frac{\partial U}{\partial Z}-\frac{V^{2}}{R} \\
=-\frac{\partial P}{\partial R}+\frac{\partial^{2} U}{\partial R^{2}}+\frac{\partial^{2} U}{\partial Z^{2}}+\frac{1}{R} \frac{\partial U}{\partial R}-\frac{U}{R^{2}} \\
\frac{\partial W}{\partial \tau}+U \frac{\partial W}{\partial R}+W \frac{\partial W}{\partial Z} \\
=-\operatorname{Gr} T-\frac{\partial P}{\partial Z}+\frac{\partial^{2} W}{\partial R^{2}}+\frac{\partial^{2} W}{\partial Z^{2}}+\frac{1}{R} \frac{\partial W}{\partial R}, \\
\frac{\partial V}{\partial \tau}+U \frac{\partial V}{\partial R}+W \frac{\partial V}{\partial Z}+\frac{U V}{R} \\
=\frac{\partial^{2} V}{\partial R^{2}}+\frac{\partial^{2} V}{\partial Z^{2}}+\frac{1}{R} \frac{\partial V}{\partial R}-\frac{V}{R^{2}}, \\
\frac{\partial T}{\partial \tau}+U \frac{\partial T}{\partial R}+W \frac{\partial T}{\partial Z} \\
=\frac{1}{\operatorname{Pr}}\left[\frac{\partial^{2} T}{\partial R^{2}}+\frac{\partial^{2} T}{\partial Z^{2}}+\frac{1}{R} \frac{\partial T}{\partial R}\right], \\
\quad \frac{\partial(U R)}{\partial R}+\frac{\partial(W R)}{\partial Z}=0,
\end{gathered}
$$

where $\operatorname{Pr}=c_{p} \mu / k$, the Prandtl number, and $\mathrm{Gr}=$ $h^{3} g \beta\left(\theta_{h}-\theta_{1}\right) / 2 \nu^{2}$, the Grashoff number. The dimensionless heat transfer rate, or Nusselt number, is then introduced as the ratio of the effective thermal conductivity to the actual thermal conductivity. Therefore,

$$
\mathrm{Nu}=\frac{1}{2}\left[\int_{Z=0}^{Z=H}\left(\frac{\partial T}{\partial R}\right)_{R=1}(d Z)\right] R_{1} \ln \left(\frac{R_{2}}{R_{1}}\right) .
$$

The pressure terms in Eqs. (1) and (2) may be eliminated by cross differentiation and subtraction to yield the vorticity transport equation

$$
\begin{aligned}
\frac{\partial \eta}{\partial \tau}+ & U \frac{\partial \eta}{\partial R}+W \frac{\partial \eta}{\partial Z}-\frac{U \eta}{R}-\frac{2 V}{R} \frac{\partial V}{\partial Z} \\
= & \text { Gr } \frac{\partial T}{\partial R}+\frac{\partial^{2} \eta}{\partial R^{2}}+\frac{\partial^{2} \eta}{\partial Z^{2}}+\frac{1}{R} \frac{\partial \eta}{\partial R}-\frac{\eta}{R^{2}},
\end{aligned}
$$

where the vorticity $\eta$ has been defined as

$$
\eta=\frac{\partial U}{\partial Z}-\frac{\partial W}{\partial R}
$$

The stream function $\varphi$ is introduced so that the continuity equation is satisfied:

$$
U=\frac{1}{R} \frac{\partial \varphi}{\partial Z} ; \quad W=-\frac{1}{R} \frac{\partial \varphi}{\partial R} .
$$

The vorticity is then expressed in terms of the stream function as

$$
\eta=\frac{1}{R}\left[\frac{\partial^{2} \varphi}{\partial R^{2}}+\frac{\partial^{2} \varphi}{\partial Z^{2}}-\frac{1}{R} \frac{\partial \varphi}{\partial R}\right]
$$

The boundary conditions for the problem are expressed in terms of stream functions, temperatures, and tangential velocities as

$$
\begin{aligned}
& \text { Thermal } \\
& \text { boundary Velocity boundary } \\
& \text { Surface conditions conditions } \\
& R=1, \quad T=-1, \quad \varphi=\frac{\partial \varphi}{\partial R}=0 ; V=\Omega \text {, } \\
& R=R_{2}, \quad T=+1, \quad \varphi=\frac{\partial \varphi}{\partial R}=0 ; V=R_{2} \Omega, \\
& Z=0, \quad \frac{\partial T}{\partial Z}=0, \quad \varphi=\frac{\partial^{2} \varphi}{\partial Z^{2}}=0 ; \frac{\partial V}{\partial Z}=0, \\
& Z=H, \quad \frac{\partial T}{\partial Z}=0, \quad \varphi=\frac{\partial \varphi}{\partial Z}=0 ; \mathrm{V}=R \Omega .
\end{aligned}
$$

The vorticity, tangential velocity, and energy equations [Eqs. (7), (3), and (4), respectively] along with the definitions of the stream function and the vorticity, and the boundary conditions then represent a condensed formal statement of the problem.

\section{FINITE DIFFERENCE APPROXIMATIONS}

In the numerical solution of a differential equation, the dependent variables are assumed to exist 
at a finite number of regularly spaced values of the independent variables known as grid points. All derivatives in the governing differential equations are replaced by finite differences involving the values of the dependent variables at the grid points. The differential equations are then reduced to a series of algebraic equations which may be solved by conventional means using a digital computer.

Once a solution for the vorticity, angular velocity, and temperature fields is known at any time (as for example, at the start of a calculation when their values are given by the initial conditions), then a solution for a short time later may be evaluated in several ways. The two-dimension implicit, alternating-direction method of Douglas, Peaceman, and Rachford $^{7-9}$ was used in this work.

Radial and vertical velocities, and the values of the vorticity on the wall (no vorticity boundary conditions are available, and, therefore, the wall vorticities cannot be calculated directly from the governing differential equations), are held constant throughout the time step at their values before the time step began. Although this procedure introduces error into the unsteady state results, this vanishes at steady state and was of no concern since only steady-state results were considered.

Once values of the vorticity at the end of the time step are known, the new stream function field must be determined. The stream function field is related to the vorticity field by the differential equation

$$
\frac{1}{R}\left(\frac{\partial^{2} \varphi}{\partial R^{2}}+\frac{\partial^{2} \varphi}{\partial Z^{2}}-\frac{1}{R} \frac{\partial \varphi}{\partial R}\right)-\eta^{n+1}=0 .
$$

Equation (11) greatly resembles a steady-state heat-conduction problem with a nonuniform generation term $(-\eta)$. Therefore, this equation was solved by converting it to the unsteady state form

$$
\frac{1}{R}\left(\frac{\partial^{2} \varphi}{\partial R^{2}}+\frac{\partial^{2} \varphi}{\partial Z^{2}}-\frac{1}{R} \frac{\partial \varphi}{\partial R}\right)-\eta^{n+1}=\frac{\partial \varphi}{\partial \tau^{1}}
$$

and integrating in "time" with the alternating direction implicit procedure until the stream function field no longer varied with time. Early experiments indicated that large "time" steps could be used so that steady state was approached rapidly-in most cases within 1-5 iterations.

Once the new field of stream functions are evaluated, the wall vorticities and velocity fields must

\footnotetext{
7 J. Douglas, J. Soc. Ind. Appl. Math. 3, 42 (1955).

8 J. Douglas and D. W. Peaceman, A.I.Ch.E. J. 1, 505 (1955).

${ }^{9}$ D. W. Peaceman and H. H. Rachford, J. Soc. Ind. Appl. Math. 3, 28 (1955).
}

FIG. 1. The model studied.
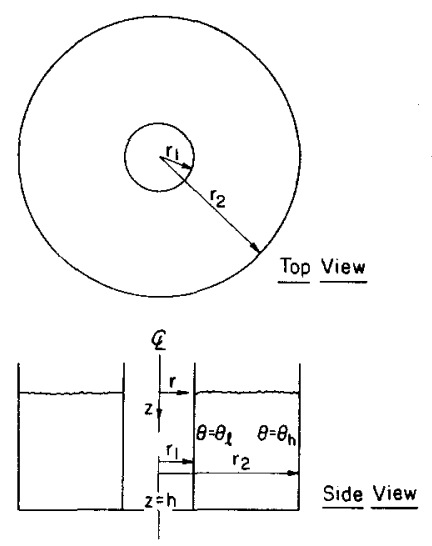

be updated. The wall vorticities were calculated from the stream function field by the technique of Churchill and Wilkes, ${ }^{10}$ and Churchill and Samuels. ${ }^{11}$

Four-point, central difference formulas were used to evaluate $U$ and $W$ for grid points not adjacent to a boundary. For grid points adjacent to a boundary, four-point noncentral difference formulas were used. A four-point difference formula was used in the evaluation of $(\partial T / \partial R)_{R=1}$ for determination of the Nusselt number.

\section{RESULTS}

A computer program for the desired calculations was written in the "MAD" computer language and the calculations were performed at the University of Michigan Computing Center.

Previous studies performed at the University of Michigan $^{10,11}$ indicated that computations of natural convection fields could be performed with Rayleigh numbers (based on $R_{2}-R_{1}$, rather than $R_{1}$ ) up to 20000 without encountering severe numerical difficulties. Since this problem is more complex than those which were previously attempted, the Rayleigh numbers investigated in this study were restricted to approximately 10000 (based on $R_{2}$ $R_{1}$ ). The Rayleigh number, $\mathrm{Ra}$, is given by $\mathrm{Ra}=$ $L^{3} g \rho \beta\left(\theta_{h}-\theta_{1}\right) / 2 \nu \kappa$, where $L$ is some characteristic length, and $\kappa=c_{p} \rho / k$, the thermal diffusivity.

Because of the limited computational time available for this study, it was necessary to restrict the number of parameters which would be studied. Since many free-convection problems of interest occur in gases whose $\mathrm{Pr}$ are of the order unity, it was decided to study only $\operatorname{Pr}=1.0$. (Previous work by this author ${ }^{11}$ indicates that for some problems in natural

${ }^{10}$ J. O. Wilkes and S. W. Churchill, A.I.Ch.E. J. 12, $161(1966)$.

${ }^{11}$ M. R. Samuels and S. W. Churchill, A.I.Ch.E. J. 13. 77 (1967). 
TABLE I. Summary of geometries studied.

\begin{tabular}{|c|c|c|c|c|c|}
\hline \multirow{2}{*}{ Case } & \multirow{2}{*}{$R_{1}$} & \multirow{2}{*}{$R_{2}$} & \multirow{2}{*}{$H$} & \multicolumn{2}{|c|}{$\begin{array}{l}\text { Number of } \\
\text { grid points }\end{array}$} \\
\hline & & & & Radial & Vertical \\
\hline Case I & 1.0 & 2.5 & 3.0 & 10 & 20 \\
\hline Case II & 1.0 & 4.0 & 1.0 & 20 & 10 \\
\hline
\end{tabular}

convection the effect of $\operatorname{Pr}$ is quite small for $\operatorname{Pr} \geq 1.0$, and therefore, the results obtained for $\operatorname{Pr}=1.0$ may apply for $\operatorname{Pr}>1$ as well.)

Two geometries, as summarized in Table I were studied, case I geometries were studied in an effort to compare the experimental results of $\mathrm{Hide}^{3}$ with values predicted by the numerical procedure; while the case II geometries were studied since they are more representative of the atmosphere of the earth.

The number of horizontal, and vertical grid spaces used in the numerical calculations are presented in Table I. Although more accurate results may be obtained with a finer grid, computational time increases as the cube of the number of grid spaces. Past experience with computations of this type $^{10,11}$ indicates that the grid sizes used in this study represent a good compromise between accuracy and computational time, when accuracy is not critical, or if machine time is limited.

Time-step sizes were chosen at the maximum value that would give stable results, and usually varied between $\Delta \tau=0.005$ and 0.01 . Steady state was generally attained at dimensionless times ranging between $\tau=1.0$, and 2.0 , and required between 8 and 12 minutes of computational time (IBM 7090 ) for a $10 \times 20$, or $20 \times 10$ grid.

Early calculations indicated that numerical difficulties were encountered at dimensionless rotational speeds $\left(\Omega=\omega r_{1}^{2} / \nu\right.$, where $\omega=$ rate of angular rotation) greater than 40 . Although it is felt that smaller grid sizes and time-step sizes could have reduced these difficulties, sufficient computational time was not available to test this hypothesis. Calculations are reported only in those instances where little, or no, numerical difficulties were encountered, and in which a steady state was attained.

TABLE II. Summary of parameters studied.

\begin{tabular}{lcccc}
\hline Case & Pr $\begin{array}{c}\text { Ra (based } \\
\text { on } R_{1}\end{array}$ & $\begin{array}{c}\text { Ra (based } \\
\left.\text { on } R_{2}-R_{1}\right)\end{array}$ & $\Omega$ \\
\hline Case I & 1.0 & 3000 & $\begin{array}{c}10100 \\
10800\end{array}$ & $\begin{array}{l}0,10,20 \\
0,10,20,40\end{array}$ \\
Case II & 1.0 & 400 & 1000 &
\end{tabular}
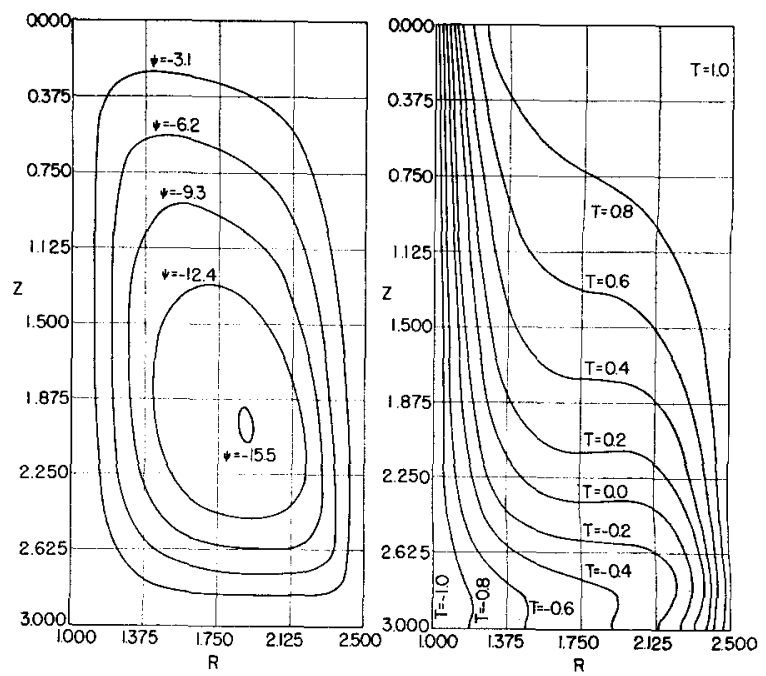

FIG. 2. Streamlines and isotherms for case $I$ with $\mathbf{\Omega}=\mathbf{2 0}$.

A complete summary of the ranges of parameters studied is presented in Table II.

\section{DISCUSSION AND RESULTS}

Steady-state streamlines and isotherms for some of the cases which were studied are presented in Figs. 2-4. In Fig. 2 a typical streamline and isotherm plot is presented for the case I geometry. From this plot, it is seen that all outward bound flow (from $R_{1}$ to $R_{2}$ ) is confined to a thin layer along the bottom surface. On the other hand, the return flow has lower velocities, and covers a much larger portion of the flow channel (height). This pattern results from the interaction of three separate causes: the geometry of the confining boundaries, the basic natural convection field, and the centrifugal and Coriolis accelerations caused by the angular rotation. The outward-bound fluid covers less than
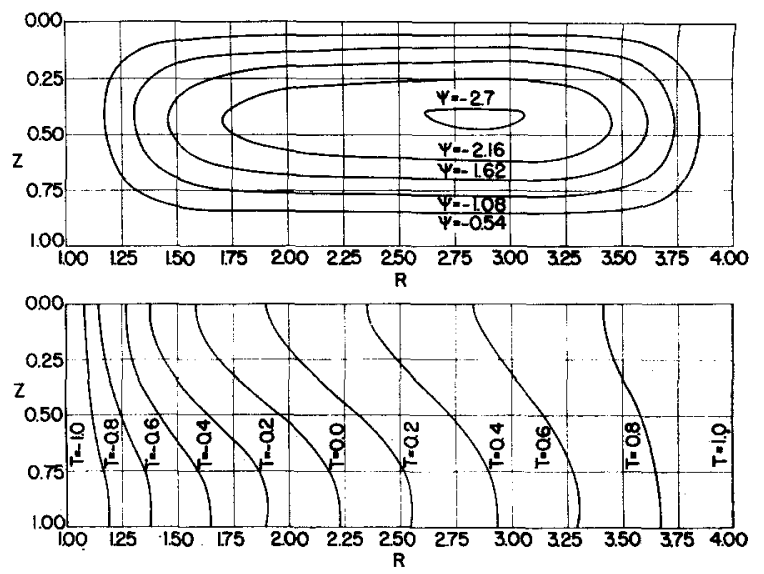

FIG. 3. Streamlines and isotherms for case II with $\Omega=0$. 

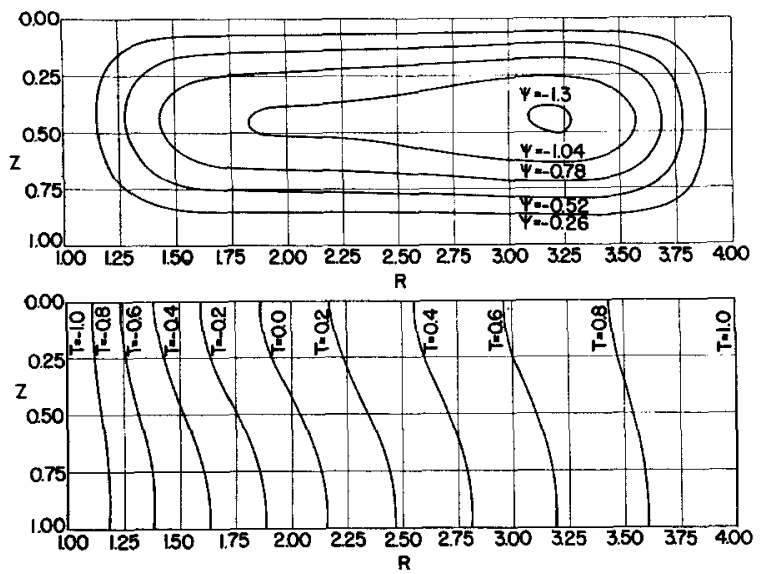

FIG. 4. Streamlines and isotherms for case II with $\Omega=10$.

haif of the flow channel in these cases because the fluid is flowing into a region of expanding flow area, and, therefore, requires less of the flow channel. The inbound fluid, however, encounters a region of decreasing area and, therefore, requires a greater portion of the flow channel. Increasing rotational speed tends to amplify this phenomenon slightly since the outbound fluid is now accelerated by centrifugal forces into a still thinner layer, while the inbound fluid encounters an even greater resistance, and tends to occupy a greater portion of the channel height.

The isotherms from the case I geometries indicate the presence of a fairly strong convective motion. The free surface on the upper boundary allows high velocities at this boundary, which in turn leads to high heat transfer rates. This is evidenced by the small distance between isotherms in the upper region near the inside boundary.

Increasing angular rotation in case I leads to

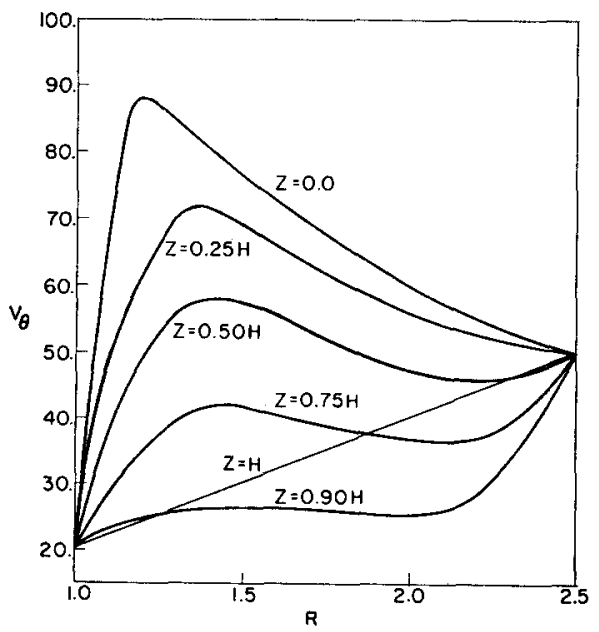

Frg. 5. Plot of tangential velocity versus radial position for case I with $\Omega=20$.
Table III. Nu for case I.

\begin{tabular}{rr}
\hline$\Omega$ & $\mathrm{Nu}$ \\
\hline 0 & 5.8 \\
10 & 5.5 \\
20 & 4.7 \\
\hline
\end{tabular}

decreased convective strength as, since the increased angular rotation causes greater flow inhibition in the inward-bound fluid. This in turn leads to lower convective heat transfer in the upper portion of the flow channel and a slight decrease in the over-all heat transfer rate. Although this phenomenon is visible in the less distorted isotherms which occur at higher rotational speeds, a more quantitative measure of its effect is seen by examination of the total heat transfer rate $\mathrm{Nu}$ as shown in Table III.

In Fig. 5, plots of the tangential velocity $V$ versus radial position are presented for several values of $Z$, at a rotational speed of $\Omega=20$ in the case $I$ geometry. Examination of these plots yields the following observations. In the upper portion of the flow channel, the fluid (which is moving inwards) has a higher tangential velocity than the fluid at the bottom surface. The fluid in the lower part of the region (which is moving outwards), on the other hand, generally exhibits a lower tangential velocity than the solid wall. These phenomena may be explained on the basis of the principle of conservation of angular momentum. Thus, the fluid in the upper portion of the region which is moving towards the axis of rotation increases its tangential velocity in a tendency to keep its angular momentum constant. The fluid in the lower part of the region on the other hand, which is moving away from the
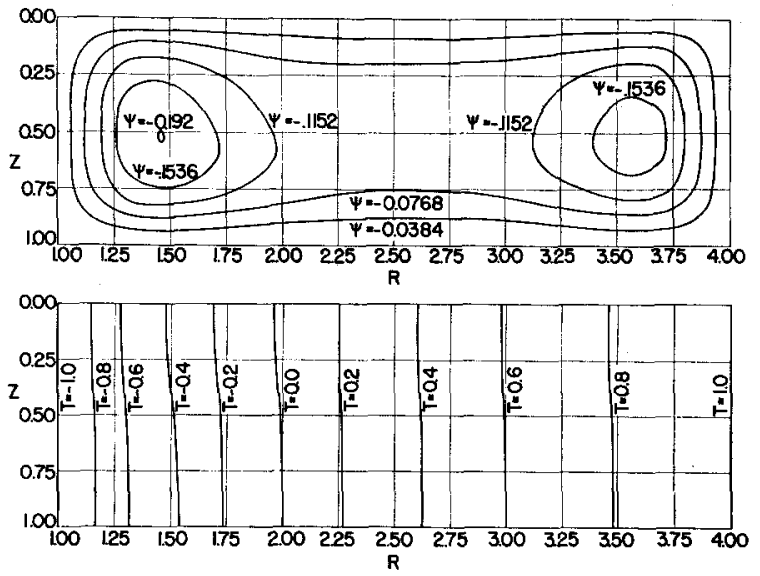

FIG. 6. Streamlines and isotherms for case II with $\Omega=40$. 
center, tends to undergo a decrease in tangential velocity. These tendencies are, however, modified by the effect of viscosity. Thus, the outward flowing fluid first experiences a slight increase in tangential velocity owing to the effect of the fluid above it, and the rotating bottom beneath it. However, viscous effects then become less important, and the aforementioned local decrease in $V$ occurs until the outside surface $R=R_{2}$ is approached. At the top surface (assumed to be a free surface), where the effects of viscosity are lowest, the fluid undergoes a large increase in tangential velocity. As the inner cylinder is approached, viscosity again becomes significant and the tangential velocity decreases rapidly to the value at the inner surface.

A somewhat similar behavior was found in the tangential velocities for case II. However, since the radial velocities for case II are significantly lower than those encountered in case I, their effect on the tangential velocity is much lower.

In Figs. 3, 4, and 6, the effect of increasing rotational speed can be seen for case II. At very low rotational speeds, a single convection cell forms. As seen in Fig. 4, this cell is quite elongated in the central region. As the rotational speed increases, the elongated section of the convection cell breaks away from the parent cell, and forms a second cell, as shown in Fig. 6.

The isotherms for case II are nearly straight vertical lines, indicating that the strength of the convective motions in these geometries is weaker than those for case I. However, this effect is to be

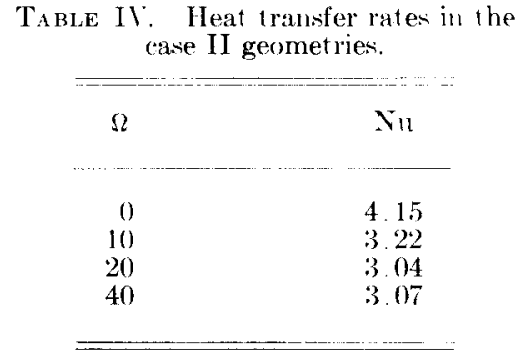

expected since the lengths of the hot and cold surfaces are smaller for case II than for case I.

In Table IV, the heat transfer rates for case II are presented. From Table IV, it may be seen that at low rotational speeds, increasing rotation causes a decrease in the $\mathrm{Nu}$, as in the case $\mathrm{I}$. However, as the rotational speed is increased still further, the heat transfer rate then increases. The increase in heat transfer in case II at high rotational speeds is apparently caused by the formation of the second convection cell. This splitting of the flow pattern tends to reduce the resistance to the inward bound flow in the upper portion of the flow channel, and leads to the increase in the heat transfer rate.

\section{ACKNOWLEDGMENTS}

The author wishes to thank Professor Chia-Shun Yih of The University of Michigan Department of Engineering Mechanics for suggesting this topic.

This work was carried out under the joint sponsorship of the National Science Foundation, and the Army Research Office in Durham, North Carolina. 